\title{
Veillée de langues endeuillées. Circonfession (1991) de Jacques Derrida
}

A Vigil of Bereaved Languages. Circumfession (1991) by Jacques Derrida

Jérémie Majorel

\section{CpenEdition}

\section{Journals}

Édition électronique

URL : https://journals.openedition.org/recherchestravaux/2991

DOI : 10.4000/recherchestravaux.2991

ISSN : 1969-6434

Éditeur

UGA Éditions/Université Grenoble Alpes

Édition imprimée

ISBN : 978-2-37747-241-3

ISSN : 0151-1874

\section{Référence électronique}

Jérémie Majorel, «Veillée de langues endeuillées. Circonfession (1991) de Jacques Derrida »,

Recherches \& Travaux [En ligne], 97 | 2020, mis en ligne le 12 novembre 2020, consulté le 21 septembre 2021. URL : http://journals.openedition.org/recherchestravaux/2991 ; DOI : https://doi.org/10.4000/ recherchestravaux.2991

Ce document a été généré automatiquement le 21 septembre 2021.

(C) Recherches \& Travaux 


\title{
Veillée de langues endeuillées. Circonfession (1991) de Jacques Derrida
}

\author{
A Vigil of Bereaved Languages. Circumfession (1991) by Jacques Derrida
}

\author{
Jérémie Majorel
}

«Dans le travail de deuil, ce n'est pas la douleur
qui travaille : elle veille. »
Maurice Blanchot, L'Écriture du désastre (Paris,
Gallimard, 1980, p. 86)
"Comment nous attarder à des livres auxquels,
sensiblement, l'auteur n'a pas été contraint?
Georges Bataille, « Avant-propos » au Bleu du ciel
(Romans et récits, Paris, Gallimard, " Pléiade »,

2004, p. 111)

1 Publié dans la collection «Les Contemporains» au Seuil en 1991, Circonfession, de Jacques Derrida est couplé avec Derridabase, de Geoffrey Bennington, qui tente la gageure de restituer point par point la philosophie de la déconstruction sans faire aucune citation. Bennington se contente de mentionner les références qui étayent en filigrane son argumentation, en prenant pour modèle le logiciel informatique et la base de données. Derridabase occupe les deux tiers principaux de chaque page. Circonfession est cantonné en bas, où se trouvent habituellement les notes ${ }^{1}$. Une ligne horizontale les sépare et un fond gris distingue nettement le texte de Derrida. Cette double bande de lecture est régulièrement interrompue par des pages illustrées et légendées. En annexe, Bennington établit une chronologie biographique, une bibliographie primaire et secondaire ainsi qu'une iconographie.

2 On peut très bien lire Derridabase avant Circonfession, ou après, en même temps, l'un sans l'autre... De même, Circonfession pourrait être analysé dans sa compétition avec Derridabase, dans son rapport aux Confessions de saint Augustin, en regard de la production derridienne antérieure et postérieure, dans son inscription au sein du genre 
de l'autobiographie et du texte de deuil... À vrai dire, ce n'est pas un texte écrit dans l'après-coup de la disparition d'un proche - la mère de Derrida en l'occurrence - mais dans l'anticipation de cette disparition longuement retardée. Le décès de Georgette Derrida survient plus d'un an et demi après la fin de la rédaction du texte. Le fils accompagne de près ou de loin sa mère qui dépérit lentement, à la merci parfois d'un coup de téléphone redouté. La mort attendue n'est paradoxalement jamais plus surprenante et désarmante. S'il est permis de forger un néologisme, il s'agirait donc moins d'un texte de deuil que d'endeuillement, un peu comme on parle d'ensommeillement ou d'ensoleillement. Si le texte de deuil forme un genre à part entière, le texte d'endeuillement est plus rare, sans doute parce que l'accompagnement par ses proches d'une personne âgée en fin de vie, selon l'expression usitée, est devenu un point encore plus sensible que sa mort même².

3 Circonfession, au singulier, est sous-titré "Cinquante-neuf périodes et périphrases écrites dans une sorte de marge intérieure, entre le livre de Geoffrey Bennington et un ouvrage en préparation (janvier 1989 - 4 mai 1990)». Derrida était alors dans sa cinquante-neuvième année. "Périodes" s'entend donc au sens littéraire et chronologique. Derrida, qui insiste habituellement sur la dissociation toujours possible d'un texte et de son contexte, a voulu cette fois-ci ancrer Circonfession dans un contexte précis. Mais rien n'est directement annoncé de son contenu sauf à déplier les potentialités du mot-valise : "circonférence » et "confession » par exemple. On n'est nullement obligé de penser d'emblée à "circoncision». Il y aurait une indication générique qui fait signe vers Rousseau - sur lequel entre autres portait De la grammatologie (1967) alors que jusqu'ici Derrida n'avait rien publié sur saint Augustin et une indication énonciative : l'aveu, s'il y en a un, ne se fera pas directement mais par détours périphrastiques.

Chaque bloc typographique est précédé d'un chiffre arabe. Il court en moyenne sur trois ou quatre bas de page. Il n'est constitué que d'une seule longue phrase : impossible de citer sans tronquer à moins de restituer la totalité de chaque bloc. Depuis les deux colonnes de Glas (1974), Derrida n'en est pas ici à sa première expérimentation où philosophie et littérature deviennent indiscernables. Les cinquante-neuf phrases offrent une apparence de continuité. En fait, ce sont des manteaux d'Arlequin, des poupées gigognes, des mosaïques, des tresses ${ }^{3} .$. Chacune ménage sa propre dramaturgie énonciative. Là où Bennington opère par déficit de citations, Derrida opte pour la surabondance. Il s'agit à chaque fois d'un montage de quatre matériaux identiques: le journal du dépérissement de la mère dans sa quatre-vingthuitième année, des extraits de carnets allant de 1976 à 1981 où Derrida projetait un roman intitulé Le Livre d'Élie, l'évocation de souvenirs liés à son enfance algérienne et des citations latines provenant des Confessions de saint Augustin. Derrida opère un compromis entre les deux colonnes de Glas et l'absence totale de marqueurs typographiques pour distinguer les différentes couches énonciatives. Ainsi, les citations latines sont en italique et un astérisque signale la traduction donnée en fin de chaque section. Derrida choisit l'« édition de la bibliothèque augustinienne, par E. Tréhorel et G. Bouissou, Desclée de Brouwer, 1962 » et non l'« édition bilingue (Garnier, 1925) dans laquelle, il y a si longtemps, [il a] découvert les prières et les larmes d'Augustin ${ }^{4}$ ». Ici le critère philologique prime sur le souci autobiographique. Les citations latines, pour qui 
ne sait pas les traduire directement, interrompent la linéarité de la lecture. Les citations issues des carnets, quant à elles, sont aussi en italique, auxquelles s'ajoutent guillemets et dates entre parenthèses, mais elles peuvent se lire dans une même coulée phrastique. La présence intermittente de l'italique donne l'impression d'une moirure typographique et d'un clignotement citationnel.

Quatre matériaux donc, comme si quatre colonnes s'étaient effondrées et que les morceaux avaient servi à bricoler plusieurs murets d'un fragile mausolée. Il n'y a aucune disposition régulière, par exemple un ordre chronologique croissant ou décroissant selon qu'on partirait du présent d'énonciation ou qu'on y aboutirait. Chaque section propose son propre montage citationnel autour d'un thème quasi musical. La phrase est habitée par un phrasé, la ponctuation s'éloigne de l'usage, comme sous le coup d'un émoi qui n'aurait pas besoin de moyen plus explicite pour se dire ou qui ne pourrait se dire autrement qu'ainsi. Période et périphrase tendent parfois vers la ritournelle dont parlent Deleuze et Guattari dans Mille plateaux :

Un enfant dans le noir, saisi par la peur, se rassure en chantonnant. Il marche, s'arrête au gré de sa chanson. Perdu, il s'abrite comme il peut, ou s'oriente tant bien que mal avec sa petite chanson. Celle-ci est comme l'esquisse d'un centre stable et calme, stabilisant et calmant, au sein du chaos. Il se peut que l'enfant saute en même temps qu'il chante, il accélère ou ralentit son allure ; mais c'est déjà la chanson qui est elle-même un saut : elle saute du chaos à un début d'ordre dans le chaos, elle risque aussi de se disloquer à chaque instant ${ }^{5}$.

Le tournant de la vie d'Augustin au jardin de Milan est lui-même opéré par l'écoute d'une « ritournelle ", ainsi que traduisent Tréhorel et Bouissou :

Et voici que j'entends une voix, venant d'une maison voisine ; on disait en chantant et l'on répétait fréquemment avec une voix comme celle d'un garçon ou d'une fille, je ne sais : «Prends, lis ! Prends, lis ! » À l'instant, j'ai changé de visage et, l'esprit tendu, je me suis mis à rechercher si les enfants utilisaient d'habitude dans tel ou tel genre de jeu une ritournelle semblable; non, aucun souvenir ne me revenait d'avoir entendu cela quelque part. J'ai refoulé l'assaut de mes larmes et me suis levé, ne voyant plus là qu'un ordre divin [...]. (Saint Augustin, Les Confessions, VIII, XII, Desclée de Brouwer, 1962, p. 29)

7 La périphrase derridienne fait le trajet inverse : elle ne fige pas la «ritournelle » en «ordre divin» mais préserve son énonciation trouble entre masculin et féminin, proximité et verticalité, enfants et Père, mutisme et Verbe, jeu et injonction, pathos et apathie... Le plus vertigineux reste comment, dans l'expérience de la lecture, dans la continuité apparente de la phrase, on glisse par fondus enchaînés d'une époque à une autre. Chaque longue phrase est une archéologie, où parfois la couche la plus antique affleure à la surface. Hypermnésie et amnésie sont l'avers et le revers d'une même maladie de la mémoire. L'Alzheimer de la mère, diagnostic que ne prononce pas Derrida mais dont il décrit les effets, atteint chaque période, entre oubli d'hier et souvenir d'autrefois : l'écriture rapièce tant bien que mal cette mémoire trouée, en épouse les soubresauts. Dans un passage de la $21^{\mathrm{e}}$ section, Derrida évoque ainsi plusieurs souvenirs liés à la vue du sang :

[...] le premier que je me rappelle avoir vu de mes yeux vu, dehors, puisque je fus et reste aveugle à celui de mon sept ou huitième jour, qui se trouve être celui de la naissance de ma mère, le 23 juillet, ce premier sang qui me vint du sexe d'une cousine, Simone, 7 ou 8 ans, le jour où la pédale d'une trottinette la pénétra par accident, Verfall, avec la première sensation fantôme, cette sympathie algique autour de mon sexe qui me conduit aux serviettes-éponges que ma mère laissait traîner, «marquées » du rouge au marron, dans le bidet, lors, je le compris si tard, 
de ses propres «periods », mais il faut synchroniser les quatre temps dans la même périphrase [...] (JD, p. 97)

8 La périphrase derridienne est un montage de temporalités hétérogènes. L'anniversaire de la mère coïncide avec la date de la circoncision, après le « sept ou huitième jour » de la naissance, qui résonne à son tour avec l'âge de la cousine, « 7 ou 8 ans ». Le sang répandu pendant la circoncision reste une tache aveugle qui hante le corpus derridien. «La première sensation fantôme, cette sympathie algique autour de mon sexe » rappelle les développements de Merleau-Ponty sur le membre fantôme ressenti par les amputés $^{6}$ et l'hypothèse de Freud sur la circoncision comme castration symbolique ${ }^{7}$. "Sympathie algique » joue sans doute aussi avec ce que le philosophe nomme dans Le Monolinguisme de l'autre (1996) sa "nostalgérie ${ }^{8 " ~ " . ~ A u ~ s p e c t r e ~ d e ~ l a ~ c i r c o n c i s i o n ~}$ répond du côté féminin la cruauté de l'accouchement, de la pénétration et de la menstruation. Ce passage nous éloigne de la tradition autobiographique inaugurée par saint Augustin. Comme les ethnologues ont pu distinguer un sacré droit et un sacré gauche, il y aurait aussi une double tradition dans l'écriture de soi. On pourrait en effet ici davantage penser à Michel Leiris9. L'auteur de La Règle du jeu (1948-1966) n'hésite pas à détailler blessures et opérations subies, ou dont il a été témoin, chez les deux sexes, sur diverses parties du corps martyr. L'accident de sa petite cousine Simone dont Derrida se souvient rappelle également certains épisodes d'Histoire de l'œil (1928) de Georges Bataille - dédicataire de L'Âge d'homme (1939) du même Leiris. Les motifs abordés définissent une mythologie personnelle propre à chacun. Derrida, comme Leiris, fait aussi le choix de ne pas suivre un fil linéaire et chronologique, de susciter au contraire une lecture tabulaire où le signifiant linguistique compte autant que les événements remémorés. C'est qu'il y a sans doute aussi en filigrane la présence de Francis Ponge, via une allusion à Signéponge (1984), essai que le philosophe avait consacré au poète, dans l'expression «serviettes-éponges » qui réfère au linge dont se servaient les femmes avant l'invention des serviettes hygiéniques, ou périodiques - sens supplémentaire de "périodes ${ }^{10}$ ". " "Marquées" du rouge au marron ", elles sont le saint suaire du bas corporel, le derrière de la face divine, la maculée conception.

Derrida insère les citations de ses carnets ou des Confessions de façon à susciter un vacillement énonciatif. La première personne du singulier devient propice aux supplémentations. Augustin, Derrida: qui ventriloque qui ? D’une manière analogue, lorsque Derrida rapporte certaines paroles de sa mère, l'énonciation vacille au profit d'un funambulisme subjectif :

[...] l'autre jour à Nice alors que je lui demandai si elle avait mal (« oui ») puis où, c'était le 5 février 1989, elle eut dans une rhétorique qui n'avait jamais pu être la sienne l'audace de ce trait dont hélas elle ne saura jamais rien, n'a sans doute rien su, et qui trouant la nuit répond à ma demande : "J'ai mal à ma mère ", comme si elle parlait pour moi, à la fois dans ma direction et à ma place, quoique dans la confusion d'apparence amnésique où elle finit ses jours la mémoire de sa mère, de sa mère à elle, lui soit des plus présentes, et qu'elle lui ressemble de plus en plus, je veux dire à ma grand-mère [...] (JD, p. 28-30)

Comme si Georgette produisait des énoncés derridiens à son insu, énoncés suscitant des interprétations plurielles. Par une inversion de la scène énonciative attendue, c'est le fils qui soufflerait ici la langue à sa mère. Derrida reste "le sujet supposé savoir » (Lacan) face à Georgette ignorante. Mais tout dépend si on porte l'accent sur "savoir » ou «supposé », comme dans la modalisation « dont hélas elle ne saura jamais rien, n’a 
sans doute rien su » ou la concession « quoique » qui revient à une interprétation plus vraisemblable de l'énoncé maternel.

11 La périphrase derridienne n'est pas seulement un entrelacement entre des époques hétérogènes et différentes instances énonciatives. Au français et au latin s'ajoutent de l'anglais, de l'allemand, de l'hébreu, du grec... Presque chaque mot ou tournure est une tour de Babel sens dessus dessous qui suscite une polysémie irréductible tant à l'étage des sonorités que de la graphie. Quand on demandait à Derrida comment définir la déconstruction, il répondait souvent sur un mode joycien: "plus d'une langue ${ }^{11}$ ". Aucune langue n'est repliée sur elle-même ou déjà constituée. Non seulement les langues se trouvent dans un rapport d'étrangeté mutuel mais Derrida garde un rapport d'étrangeté à l'égard de chacune en elle-même. Circonfession revient à plusieurs reprises sur l'épreuve traversée par un juif d'Algérie naturalisé français depuis le décret Crémieux de 1870, destitué de la citoyenneté française sans que l'Algérie ne soit à proprement parler occupée par les nazis, puis rendu à cette citoyenneté :

[...] j'ai mis, moi, misé ce mot pour «mot », «pour » celle, ma mère, qui serait la dernière, et ma famille, à se retrouver dans ce que j'écris là, moi, toujours moins reconnaissable dans ma famille que dans mon pays, dans mon pays qu'en Europe, en Europe que partout ailleurs, si bien que je ne me prive pas, moi qu'on appelait «le sauvage » ou la « tête carrée », de parler latin, de vous obliger à réapprendre le latin pour lire sA, moi, au travail, le peu de latin que je sache pour avoir commencé à l'apprendre quand Vichy l'avait rendu, je crois, obligatoire en sixième juste avant de me foutre à la porte du lycée au nom latin du numerus clausus en nous retirant la citoyenneté française [...] (JD, p. 177-178)

Dans l'Algérie coloniale, le latin était obligatoire dès la classe de sixième mais l'arabe était une langue étrangère facultative. Pour Augustin lui-même, natif de ce qui correspond actuellement à Souk Ahras en Algérie, le latin n'était pas encore une langue chrétienne mais celle d'un Empire romain et d'une culture classique en voie de dislocation. La Bible n'avait pas encore été entièrement traduite par saint Jérôme. Augustin ne lisait pas l'hébreu et comprenait avec difficulté le grec. Dans la préface à sa nouvelle traduction des Confessions, Frédéric Boyer définit ainsi le geste de l'évêque d'Hippone :

Il couture littéralement son propre texte de citations entières avec lesquelles il se fait parler et penser. [...] Cette sorte de pieuse et littéraire ventriloquie lui est propre. [...] On ne dira pas assez combien il invente sa langue, transgresse les genres anciens, rhétorique, philosophique ou exégétique, récupère discours, prières, invocations et hymnes pour rédiger une narration complexe et ouverte ${ }^{12}$.

Derrida reprend à sa manière cette opération rhapsodique en utilisant Augustin comme celui-ci s'était servi des psaumes. Lui aussi «invente sa langue ", n'ayant de rapport d'évidence avec aucune. Ce rapport d'étrangeté à la langue, à toute langue, sensible dans son écriture par une tension incessante vers l'idiome et une admiration peu commune à l'égard des traducteurs pour lesquels ses textes sont autant de défis, irrite certains et en séduit beaucoup, tout autant sans doute que l'entreprise de déconstruction des grands systèmes philosophiques occidentaux.

Circonfession offre maints exemples d'étonnement par rapport à un mot ou à un tour de la langue française ou d'une autre langue, dénaturalisant ainsi les évidences et débusquant les impensés. C'est un des effets critiques de la périphrase. Le nom de ce 
trope présuppose que la phrase tourne autour de ce qui pourrait être directement dit en un seul mot. Ce trope est donc traditionnellement régi par l'illusion d'une coïncidence possible entre mot et chose. En conséquence, on a pu le condamner comme préciosité, mondanité, devinette gratuite, sublimation ridicule de la finitude, perte de temps en regard des exigences communicationnelles... Michel Deguy, dans une étude sur "Phrase, périphrase, paraphrase», résume les soubassements de cette condamnation : "Le bref - la "concision" - renvoie au préjugé de l'univocité, c'est-àdire de la préférabilité - invincible parce que "métaphysique" - de l'un sur le multiple. Préjugé de l'être-un ; croyance qu'il y aurait "une chose chaque fois" et un mot pour une chose ${ }^{13}$.» La périphrase derridienne prend acte qu'il n'y a jamais véritablement de mot propre ou impropre. Elle déconstruit en acte «le vocable cru » - il s'agit ici des tout premiers mots de Circonfession : parler crûment et sans détours du dépérissement de sa mère, parler de façon à être cru par quelqu'un à défaut de Dieu et parler avec des mots équivalents au morceau de prépuce sanglant ${ }^{14}$. Derrida n'aura jamais été aussi impudique que dans ce texte, qui s'oppose ainsi à la présentation monographique, abstraite, opérée par Bennington au-dessus ${ }^{15}$.

La périphrase derridienne revivifie les catachrèses qui sont le lit inaperçu de toute langue. Elle se tient au plus près d'une exigence à la fois philologique et philosophique, un amour de la langue qui se méfie de l'évidence des langues, des impensés véhiculés par telle expression communément admise ou émanant de certaines institutions sociales. Parfois Derrida utilise l'italique pour rendre une expression française aussi distante que du latin. Dès le premier chapitre, il s'arrête ainsi sur " "trouver la veine" " et " "prise de sang" ». Ce ne seront pas les seuls éclats d'un discours médical implantés dans ses phrases. Il cite également à plusieurs reprises ce constat à propos de l'évolution des "escarres » - «nécrose de la peau et des tissus sous-jacents, formant une croûte noire puis un ulcère, survenant surtout chez les personnes alitées " (Larousse) - de sa mère : " "ils sont beaux" » (p. 76 et p. 191, l'infirmier se trompe de genre).

16 Ni la catégorie du maternel ni celle du vernaculaire ne sont opérantes pour qui se sent exclu du français sans avoir jamais été inclus dans l'arabe ni vouloir se réfugier dans l'hébreu. Pour préparer sa bar-mitzva, Derrida fait semblant d'apprendre :

[...] je fis ma « communion » en fuyant la prison de toutes les langues, la sacrée dans laquelle on voulait m'enfermer sans m'y ouvrir, la séculaire dont on marquait qu'elle ne serait jamais la mienne, mais cette ignorance est restée la chance de ma foi comme de mon espérance, de mon goût même pour le " mot ", le goût pour les lettres [...] (JD, p. 240)

17 Il déjoue ici l'opposition entre langue sacrée et langue séculaire, comme s'il transférait le sacré dans le séculaire, ou considérait que la langue n'a pas d'autre transcendance que les mots et les lettres qui la constituent, approche qu'il faudrait confronter à celle de la kabbale. Il ne circoncit pas ses fils ni ne fait de «mariage endogame» (p. 87) ${ }^{16}$. Cela n'empêche pas Circonfession d'être parsemé d'allusions à la Brit milah - circoncision -, au mohel - celui qui l'exécute -, à la mezizah - une manière de procéder où le rabbin prend le pénis incisé de l'enfant dans sa bouche-, ainsi qu'au Pardès - équivalent judaïque du paradis et de l'exégèse. 
est en fait un patient décryptage du corps blessé, entaillé, incisé, écrit, celui de Derrida à peine né ou de sa mère sénescente, où peut se lire le signe d'une appartenance religieuse ou d'un symptôme médical, voire les deux en même temps. Le chapitre 56 débute ainsi :

Les escarres se rouvrent, me dit ma sœur au téléphone, celle du sacrum en particulier, je ressens l'impatience ambiguë de tous, la culpabilité à vif autour de ce signe de vie où le dedans paraît encore et le sang qui proteste et la peau qui ne veut pas se fermer enfin sur son silence, comme un linceul propre, comme une paupière de sommeil [...] (JD, p. 245)

$19 \mathrm{Au}$ lieu de ces détails liés au bas corporel, la famille voudrait sublimer fin de vie et vieillissement. La phrase réalise en partie cette aspiration en mêlant abstrait et concret - «culpabilité à vif » comme la blessure - et en filant une métaphore à la manière d'une suture: "paupière de sommeil» dit ainsi les yeux de la morte rituellement refermés, le déchirement de la peau cicatrisée et la pierre tombale scellée. En même temps, on ne peut s'empêcher de percevoir dans cette escarre au sacrum lue sur le corps de sa mère un punctum propre à Derrida : une blessure liée au sacré, les séquelles de sa circoncision, un rapport complexe à sa judéité ${ }^{17}$.

Le Livre d'Élie devait tourner autour du motif de la circoncision et être nourri de toute une documentation religieuse, culturelle et anthropologique. Élie est le prénom sacré et caché de Derrida, "Jacques" étant quant à lui la francisation de Jackie ${ }^{18}$. Dans Circonfession, aucun nom n'est encore gravé dans le marbre. Derrida utilise souvent l'abréviation «G. ", ce qui peut provoquer une confusion entre Geoffrey et Georgette. Le propre frère de Georgette raccourcissait parfois affectueusement son prénom en «Geo» (p. 24, p. 217-218) et Derrida surnomme Bennington «Geoff». Son dessein est d'échapper dans sa vie et dans son œuvre à toute détermination matricielle, qu'elle soit génétique ou interprétative, philosophique ou maternelle. Saint Augustin est lui aussi abrévié «sA », ce qui peut renvoyer à l'auteur des Confessions ou au savoir Absolu de Hegel. "Maman » (p. 71), "Geo», "Georgette ", "Esther " avec ou sans h comme « escarre» (p. 92), «Georgette Sultana Esther» (p. 70), le nom complet finit par être donné : «Sultana Esther Georgette Safar Derrida »(p.174). Prénoms au pluriel, second ou sacré, nom de jeune fille, nom d'épouse, chacun est la crypte variable d'une biographie possible au croisement de l'intime et du politique, du légendaire et de l'historique. Seul le patronyme reste invariable alors même que Derrida n'a jamais été très disert sur son père Aimé et aura entrepris la déconstruction de ce qu'il appelle le "phallogocentrisme" dès les années 1970 - non sans succès auprès d'un grand nombre de féministes américaines. Outre le dépérissement de sa mère, Benoît Peeters nous apprend que « [1]'année 1989 est marquée par un autre choc, qui affecte Derrida beaucoup plus qu'on pourrait le croire ${ }^{19} "$ : la publication de la thèse de son fils Pierre chez Minuit, Guillaume d'Ockham, le Singulier, sous le nom de sa grand-mère maternelle Alféri ${ }^{20}$. Georgette elle-même ne se souvient plus du prénom de son fils et n'a jamais voulu lire un de ses livres. Elle perd également peu à peu la vue. Pendant plusieurs jours d'affilée, Derrida souffre à son tour d'un curieux symptôme :

[...] et voici qu'aujourd'hui (29-6-89) je me téléphone à G. comme à Dieu, une heure avant de glisser mon corps étendu sur le dos dans la capsule tombale d'un scanner de Neuilly pour lui raconter ce que j'écris tout contre lui et lui annoncer que depuis quelques jours mon visage se voit défiguré par une paralysie faciale qui tient mon œil gauche fixe ouvert comme un cyclope à bille de verre, vigilance imperturbable du mort, la paupière tendue par la barre verticale d'une cicatrice intérieure, an invisible scar, lui dis-je, et la bouche tordue dont l'eau retombe un peu sur le menton 
quand je bois, me rappelant à ma mère chaque fois que, d'une main soutenant sa tête, je verse l'eau dans sa bouche plutôt qu'elle ne la boit, ecce ubi sum ! flete mecum, et pro me flete ${ }^{*}[. .$.$] (JD, p. 89-90)$

21 La traduction est donnée plus loin : "“Voilà où j'en suis! Pleurez avec moi et pleurez pour moi." (X, XXXIII, 50)» (p. 91) Le latin d'Augustin permet ici à Derrida d'exprimer ce qu'il semble s'interdire en français : l'émotion, les larmes, les prières qu'un athée peut formuler sans vraiment y croire en de pareilles circonstances. Dans Les Confessions, cette prière conclut un développement où Augustin avoue à Dieu sa fascination pour les plaisirs liés au sens de l'ouïe, notamment lorsqu'il écoute les psaumes de David chantés en chœur au point d'en oublier les paroles au profit de la seule musicalité. Derrida l'insère dans son propre contexte énonciatif. Il rapporte une conversation téléphonique avec Bennington. Il s'attarde sur un examen médical nécessité par sa paralysie faciale du côté gauche. Cette mésaventure physiologique ne va pas sans une identification mélancolique à la mère alitée, comme en témoigne la métaphore de la «capsule tombale ", les échos de "scanner » et «scar » avec une « escarre » tacite, l' « œil gauche fixe ouvert » comme celui de la morte en puissance dont le regard ne semble déjà plus rien percevoir et une difficulté commune à satisfaire un besoin physiologique aussi simple que de boire de l'eau. Tout ceci dans la fluidité d'un phrasé qui passe de la bouche aux yeux, de l'eau aux larmes, de ce qui s'ingère à ce qui s'exprime.

Derrida nomme donc directement le corps vieillissant et immobilisé de sa mère. Il fait face au «spectacle nu d'une blessure photographiée - l'escarre cautérisée par la lumière de l'écriture, à feu, à sang mais à cendre aussi » (p. 55). L'escarre est le punctum, le détail poignant, qui désorganise le studium d'un corps déchu de sa photogénie. Elle est ce point critique d'un corps ni tout à fait vivant ni tout à fait mort, un corps de mourante. Les allitérations en sifflantes sont ponctuellement interrompues par « cautérisée »: la ligne sinusoïdale se brise provisoirement, pas encore définitivement. Mais la métaphore photographique est aussi ici médicale : radio et laser, examens, traitements, voire "acharnement thérapeutique» (p.152). L'écriture est un soin ambigu, un pharmakon, ce que suggère la reprise décalée de la locution «à feu et à sang ", de signification guerrière et sans merci à l'origine. Feu la cendre, écrivait Derrida en 1987. Le passage est fulgurant entre "feu » et "cendre " à travers «sang ", par propagation sonore d'une crémation.

Circonfession faisait donc partie d'une monographie publiée initialement dans la collection de Denis Roche. Derrida, mais aussi Wittgenstein, y côtoyait poètes, dramaturges et romanciers. Son texte, réédité en 2008, est toujours contemporain, moins au sens de préfiguration de mouvements littéraires dominants aujourd'hui que tout contre eux : les narrations documentaires par la pratique du montage citationnel, générique et photographique ; l'autofiction par ce que Guillaume Artous-Bouvet préfère nommer à son propos "hétérofiction ${ }^{21}$ ». Le philosophe allie comme rarement un dispositif expérimental, un rapport d'étrangeté à la langue, une nécessité intime et des enjeux historiques, politiques et théologiques. Sa contemporanéité ne se réduit pas au présentisme sans lorgner pour autant uniquement vers le passé. Derrida n'hésite pas à citer en latin Les Confessions mais détourne allégrement chaque citation. Il les traite comme un matériau, dans une attitude davantage pragmatiste qu'exégétique. De même, il expérimente un montage syntaxique sans verser dans la gratuité formaliste mais en 
obéissant à une nécessité valable cette fois seule: «chaque fois unique, la fin du monde ».

\section{NOTES}

1. Dans L'Invention du commentaire. Augustin, Jacques Derrida (Paris, PUF, "Écriture ", 2000), Bruno Clément écrit : « La position infrapaginale, comme ancillaire, de son texte rappelle celle de ces gloses médiévales écrites par les moines copistes dans les marges des manuscrits, de ces commentaires avertis, érudits, et parfois égarants, quelquefois même peut-être fautifs (et alors rectifiés par d'autres moines, lisant parfois longtemps après eux) qu'ils apposaient, avec une écriture différente, plus petite, au bas de ces parchemins que d'autres enluminaient, pour qu'en jaillisse en effet la lumière. » (p. 105)

2. Voir dans ce volume l'étude de Laurent Demanze sur Devant ma mère (2007) de Pierre Pachet. Sur le texte de deuil, voir l'essai de D. Carlat, Témoins de l'inactuel. Quatre écrivains contemporains face au deuil, José Corti, coll. «Les Essais ", 2007. Glas (1974), Fors (1976), Feu la cendre (1987), Mémoires - pour Paul de Man (1988), Spectres de Marx (1993), Adieu - à Emmanuel Levinas (1997), Demeure - Maurice Blanchot (1998), Donner la mort (1999), Chaque fois unique, la fin du monde (2003) ... : l'œuvre de Jacques Derrida peut être entendue comme un immense thrène qui entrelace intime et politique, amitié et pensée, apocalypse et histoire, abolition des traces et survivance indéfectible. Cf. D. Farrell Krell, The Purest of Bastards: Works of Mourning, Art, and Affirmation in the Thought of Jacques Derrida, University Park, Pennsylvania State University Press, 2000 ; J. Rogozinski, Faire part. Cryptes de Derrida, Paris, Lignes, 2005 ; A. Jdey, «Derrida, l'autre et le deuil. Vers une herméneutique des larmes ", dans Les Larmes modernes, mélanges coordonnés par F. Toudoire-Surlapierre et N. Surlapierre, Paris, Éditions L'Improviste, 2010, p. 151-169.

3. Bruno Clément parle de "tresse» (L'Invention du commentaire, ouvr. cité, p. 97) et de « mosaïque d'altérité » (ibid., p. 109).

4. G. Bennington et J. Derrida, Jacques Derrida [1991], Paris, Seuil, coll. « Les Contemporains », 2008 , p. 17. Les références à cet ouvrage se feront dans le corps du texte désormais, sous l'abréviation JD.

5. G. Deleuze et F. Guattari, Mille plateaux, Paris, Minuit, 1980, p. 382.

6. Voir M. Merleau-Ponty, Phénoménologie de la perception, Paris, Gallimard, coll. «Tel », 1945, p. 90-105.

7. Voir S. Freud, Abrégé de psychanalyse [1940], traduit de l'allemand par A. Berman, neuvième édition revue et corrigée par Jean Laplanche, Paris, PUF, 1978, p. 60-62. Dans d'autres passages, Derrida utilise le verbe « remembrer» (p. 72 par exemple) : terme vieilli et littéraire pour «se souvenir ", mot qui rappelle l'anglais remember mais aussi, littéralement, recoller un membre sectionné.

8. Voir J. Derrida, Le Monolinguisme de l'autre, Paris, Galilée, 1996, p. 86. Étymologiquement, «nostalgie » signifie douleur de ne pouvoir retourner chez soi. Peut-être que la prédilection de Derrida pour le mot-valise est liée à une hantise de l'exil, une manière de suturer la coupure.

9. Voir G. Poitry, Michel Leiris, dualisme et totalité, Toulouse, Presses universitaires du Mirail, «Cribles », 1995.

10. L'éponge est un des motifs de Circonfession (voir p. 49, p. 94-96 et p. 98). Ponge est mentionné à plusieurs reprises. 
11. Rappelons que Derrida est aussi l'auteur d'Ulysse gramophone. Deux mots pour Joyce, Paris, Galilée, 1987.

12. Fr. Boyer, « Préface », dans saint Augustin, Les Aveux, Paris, P.O.L., 2013, p. 30-31.

13. M. Deguy, «Phrase, périphrase, paraphrase », Poétique, $n^{\circ} 117$, février 1999, p. 71.

14. Bruno Clément résume ainsi : «Le rêve - très ancien rêve - est formulé dès les premières lignes de Circonfession, dès la première phrase : rêve d'un mot, un seul, proféré sans précaution ni périphrase, presque sans phrase, rêve de ce que Jacques Derrida appelle, en référence au petit morceau de chair vive et sanglante rituellement retranché, "le vocable cru" (premiers mots). » (L'Invention du commentaire, ouvr. cité, p. 85)

15. B. Peeters, Derrida, Paris, Flammarion, "Grandes Biographies », 2010 : «À côté d'un corpus dont le critique "n'a pas gardé un seul fragment intact", il réintroduit le corps, y compris son propre pénis. Au moment même où l'exposé méthodique de Geoffrey Bennington fait de lui un philosophe quasi recevable, voici que Derrida s'emploie à déconstruire de l'intérieur l'ouvrage qui lui est consacré. » (p. 495) Voir aussi J. Derrida, L'Animal que donc je suis, Galilée, 2006.

16. Bruno Clément perçoit ainsi un chiasme entre Derrida "juif plus ou moins renégat » et Augustin « chrétien converti » (L'Invention du commentaire, ouvr. cité, p. 7).

17. Voir J. Cohen et R. Zagury-Orly (dir.), Judéités. Questions pour Jacques Derrida, Paris, Galilée, 2003. Voir également la partie «Le Juif » de Derridabase (p. 242-246). À propos du vocabulaire de Barthes que je viens d'utiliser, Bennington avait en tête Roland Barthes par Roland Barthes (1975) en proposant à Denis Roche, directeur de la collection « Les Contemporains » au Seuil, cet ouvrage hybride. Derrida avait proposé une lecture de La Chambre claire (1980) en hommage au critique disparu, «Les morts de Roland Barthes », dans Poétique en 1981. C'est ce texte qui sera repris en ouverture de Chaque fois unique, la fin du monde (2003). Les illustrations qui scandent la lecture et le journal du dépérissement de la mère suggèrent immanquablement le rapprochement de Circonfession avec La Chambre claire, fût-ce pour mieux percevoir les décalages.

18. Bruno Clément observe que «le livre projeté [...] aurait raconté comment [le nom d'Élie] fut choisi, pourquoi il ne fut pas porté, comment il fut redécouvert " (L'Invention du commentaire, ouvr. cité, p. 84).

19. B. Peeters, Derrida, ouvr. cité,p. 498.

20. Sur les enjeux de ce qui précède, voir C. Burgelin, Les Mal Nommés. Duras, Leiris, Calet, Bove, Perec, Gary et quelques autres, Paris, Seuil, « La Librairie du XxI ${ }^{\mathrm{e}}$ siècle », 2012.

21. Voir G. Artous-Bouvet, "Le supplément de fiction : Derrida et l'autobiographie », Littérature, $\mathrm{n}^{\circ} 162$, juin 2011, p. 102-116.

\section{RÉSUMÉS}

Il s'agira dans cette étude d'entrer dans la chair d'un texte de Jacques Derrida, Circonfession (1991), qui tourne autour du dépérissement de sa mère vieillissante. Comment les choix formels déroutants adoptés par Derrida dans ce texte, qui rejoint par ailleurs la tradition autobiographique, ont-ils été contraints par cet endeuillement? C'est le corps du philosophe tout autant que celui de sa mère qui s'écrit ici - en « plus d'une langue ».

This study aims to enter into the flesh of a text written by Jacques Derrida, Circumfession (1991), dealing with the decay of his aging mother. How do the confusing formal choices adopted by Derrida in this text, which is also part of an autobiographical tradition, have been influenced by 
mourning? It's the body of the philosopher as much as his mother's that is written here-"more than a language".

INDEX

Mots-clés : Derrida, Circonfession, langue, périphrase, corps.

Keywords : Derrida, Circumfession, language, periphrasis, body.

\section{AUTEUR}

JÉRÉMIE MAJOREL

Jérémie Majorel est maître de conférences à l'université Lumière Lyon 2, membre du laboratoire Passages XX-XXI (EA 4160). Il a publié notamment Maurice Blanchot. Herméneutique et déconstruction (Honoré Champion, 2013). 